\title{
Pulmonary Rehabilitation throughout the Weaning from Mechanical Ventilation for Complete Cervical Spinal Cord Injury: A Case Report
}

\author{
Bin Zhang ${ }^{1}$, Hongying Jiang ${ }^{1, *}$, Chenxi Zhang ${ }^{1}, \mathrm{Yi} \mathrm{Li}^{1}$, Zhanqi Zhao ${ }^{2,3, *}$
}

${ }^{1}$ Department of Respiratory

Rehabilitation Centre, Beijing

Rehabilitation Hospital of Capital

Medical University, Beijing, P. R. China

${ }^{2}$ Department of Biomedical Engineering,

Fourth Military Medical University, Xi'an,

P. R. China

${ }^{3}$ Institute of Technical Medicine,

Furtwangen University,

Villingen-Schwenningen, Germany

\section{*Correspondence}

zhanqi.zhao@hs-furtwangen.de

(Zhanqi Zhao)

6jhy@163.com

(Hongying Jiang)

\begin{abstract}
The aim of this report was to present a tailored program of weaning from mechanical ventilation successfully. This program was developed for the ventilator-dependent patient with spinal cord injury (SCI) and especially targeting the respiratory muscles. A 48-year old male had a car accident on 25 May 2019 and his limb muscle strengths were severely impaired. He was diagnosed with complete cervical spinal cord injury (level C4), difficult-to-wean. Electrical impedance tomography was used to direct the rehabilitation program. Besides, passive activities (stretching of the intercostal muscles, bed tilting, and passive movement of the scapula and the proximal end of the upper limbs) and active training (various diaphragmatic facilitation techniques) were applied for inspiratory muscle training. The patient was successfully weaned from mechanical ventilation and discharged from the hospital. The individualization of rehabilitation program was demonstrated before, during, and after weaning for the SCI patient. Rehabilitation program especially targeting the respiratory muscles would facilitate the weaning process for complete spinal cord injury.
\end{abstract}

\section{Keywords}

Spinal cord injury, Respiratory muscles training, Weaning from mechanical ventilation, Rehabilitation program, Electrical impedance tomography

\section{Introduction}

Mechanical ventilation is used to save lives in the intensive care units (ICU). However, it could also cause serious complications such as ventilator-induced lung injury and diaphragm atrophy. Successful weaning from mechanical ventilation is crucial since patients with prolonged weaning from mechanical ventilation are often associated with a higher mortality rate, especially for patients with complete spinal cord injury (SCI) [1]. Different causes might be accountable for weaning failure. For patients with cervical SCI, the main causes are partial denervation of the diaphragm, paralysis of the thoracic and abdominal walls [2]. Respiratory complications are the leading cause of morbidity and mortality in patients with cervical SCI [3].

In this report, we presented a case in which a rehabilitation program was developed to facilitate the weaning process specifically targeting the respiratory muscles.

\section{Case reports}

A 48-year old male had a car accident on 25 May 2019 and his limb muscle strengths were severely impaired (right upper limb graded 3, left upper limb graded 1, and lower limbs graded 0 according to the ordinal Medical Research Council scale [4]). X-ray and CT examination indicated C5 vertebral slippage, vertebral appendices fracture and spinal stenosis; T12 vertebral body and appendices and L1 vertebral body fracture. The surgery was performed two days after the accident (front and rear cervical fusion and internal fixation, lumbar fracture reduction and bone graft fusion and internal fixation). Extubation was performed after surgery when he passed the spontaneous breathing trial (SBT). However, the muscle strength of both upper limbs was reduced to 0 three days after surgery, accompanied by chest tightness and shortness of breath. Chest CT showed atelectasis and pleural effusion at the lower lobes of both lungs (Fig. 1A). Due to sudden dyspnea and loss of consciousness occurred 8 days after the surgery, the patient was transferred to our department in Beijing Rehabilitation Hospital of Capital Medical University.

The patient was conscious but uncooperative to physical examinations, limb muscle strength graded 0 at the admission. His body temperature was $36.6^{\circ} \mathrm{C}$, heart rate 80 beats $/ \mathrm{min}$, respiratory rate 28 beats $/ \mathrm{min}$, blood pressure $92 / 55 \mathrm{mmHg}$, $\mathrm{SaO}_{2} 80 \%$. Blood gasses analysis indicated a type 2 respiratory failure ( $\mathrm{pH} 7.17, \mathrm{PaO}_{2} 74 \mathrm{mmHg}$ with $\mathrm{FiO}_{2} 0.6, \mathrm{PaCO}_{2}$ $92 \mathrm{mmHg}, \mathrm{HCO}_{3}-33.9 \mathrm{mmol} / \mathrm{L}$ ). The thickness of the diaphragm measured with ultrasound at the end of expiration was $0.17 \mathrm{~cm}$ and diaphragmatic activity $4 \mathrm{~cm}$. The neurological 

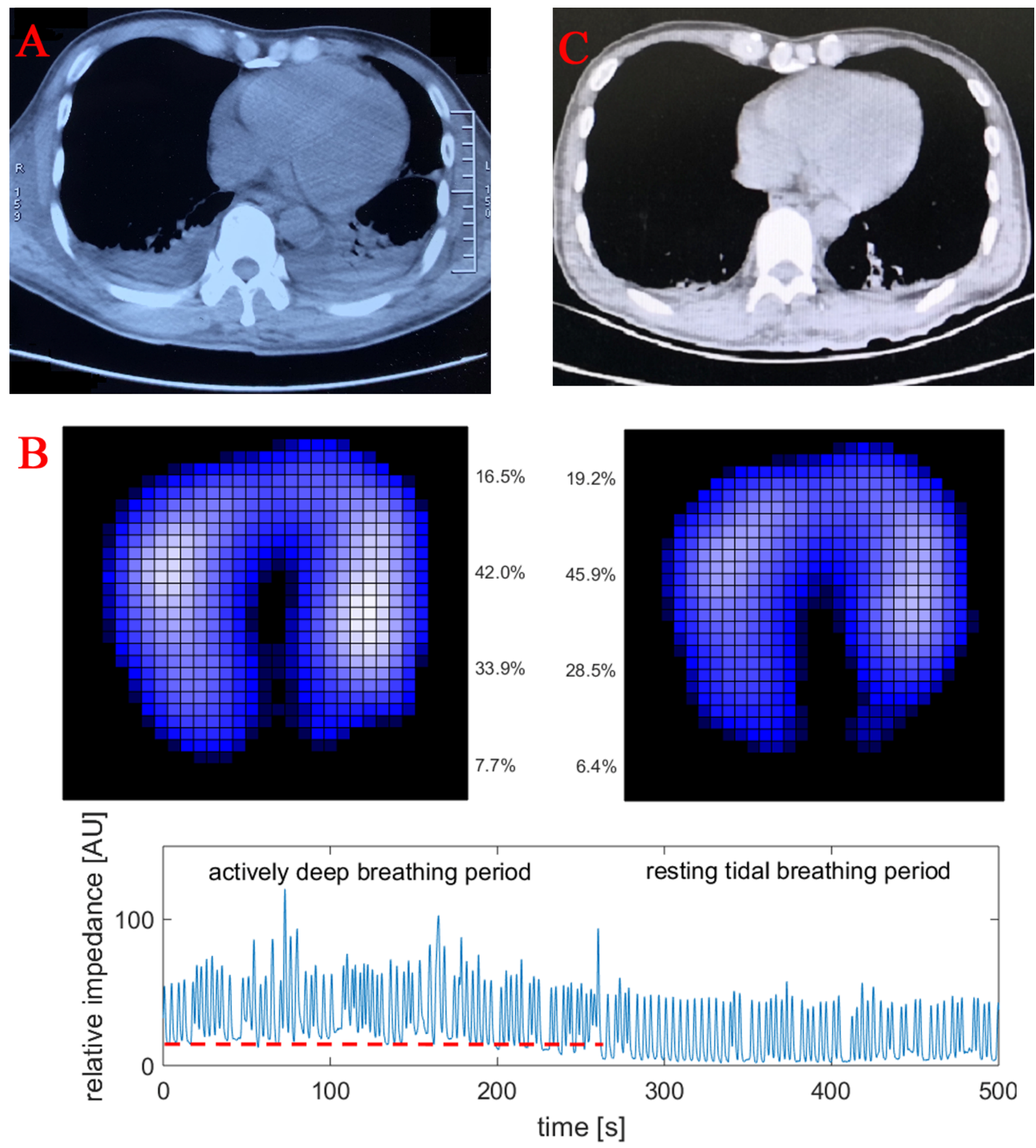

F I G U RE 1. CT and EIT measurements of the patient.

(A) Chest CT before admission indicated atelectasis and pleural effusion at the lower lobes of both lungs. (B) EIT images showing tidal variation during actively deep breathing (left) and resting tidal breathing (right) period. Light blue depicted regions with high ventilation. Percentage indicated ventilation distribution in ventral and dorsal regions along the gravity axis. Actively deep breathing improved end-expiratory lung volume (bottom figure, red dashed line). (C) CT image after weaning demonstrated significant improvement of atelectasis and pleural effusion.

level of injury was C4, American Spinal Injury Association Impairment Scale graded A. Paradoxical breathing was observed. The maximal inspiratory pressure (MIP) was 13.88 $\mathrm{cmH}_{2} \mathrm{O}$. Although the patient's condition improved after treatment with anti-infection, nebulization and eliminating phlegm,
SBT failed repeatedly. Re-examination of the documents we suspected that the causes of acute respiratory failure were a progression of respiratory muscle weakness, poor sputum drainage, and postoperative spinal cord edema. 


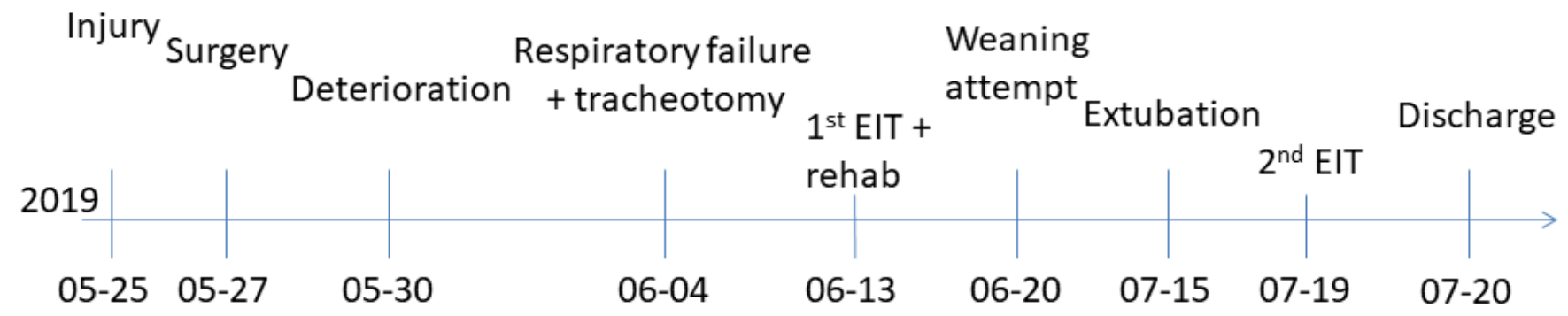

F I G U R E 2. Time course of the case highlighting the time points of the major events.

Based on the examination with electrical impedance tomography (EIT), it was noted that although the ventilation was mainly distributed to the non-dependent regions, actively deep breathing improved end-expiratory lung volume and ventilation redistributed towards the dorsal regions (Fig. 1B). We considered that the patient would benefit from an individualized pulmonary rehabilitation program. Respiratory muscle training consists of passive activities and active training, which were conducted sequentially. The passive activities of the respiratory muscles included stretching of the intercostal muscles, bed tilting 50 degree and passive movement of the scapula and the proximal end of the upper limbs, twice daily for 10 minutes each time (MOTOmed viva2, RECK-Technik, Betzenweiler, Germany). Strength and endurance training on the diaphragm were performed afterward with various diaphragmatic facilitation techniques [5]. The initial maneuver was to use the thumbs of the therapist to slide outward from the sternum stem along the lower edge of the tenth rib and to apply pressure during exhalation. Pressure was applied to the outside of the abdomen with the other fingers during inhalation to improve the rebound ability of the diaphragm (8-10 times/group, 3 groups, 3 minutes rest between groups; once every morning and afternoon). When the patient's diaphragm rebound ability improved and he began to wean, the maneuver was slightly modified: the therapist pressed down along the lower edge of the tenth rib with the thumbs to produce appropriate resistance when the patient inhaled. The intensity of the applied resistance was subjective and increased gradually: The patient was able to complete deep inhalations but would feel tired after one group of breathing (10-12 times/group) and was able to recover within the given resting time ( 3 groups, 3 minutes rest between groups; once every morning and afternoon). Inspiratory muscle training (IMT) was conducted using POWERbreathe $\mathrm{K} 5$ with live feedback and adaptation to training requirements (POWERbreathe International Limited, Warwickshire, UK). The rehabilitation program was performed during the whole hospitalization period (from admission 2019-6-13 to discharge 2019-7-20).

For clearance of airway secretion, a manual vibration maneuver was used at the beginning in the lateral position during expiration. It involved a gentle, high-frequency force to produce a vibratory pressure to the chest wall over the involved lung segment. The duration and number of replication depended on the amount and texture of the patient's secretions. At a later stage after implementation of tracheostomy speaking valve, the upper airway integrity was simulated and anatomical end-expiratory pressure is recovered [6]. The active cycle of breathing technique was then performed to remove tracheal secretions.

Finally the patient passed SBT and weaned from ventilator successfully. High-flow nasal cannula oxygen therapy was applied subsequently to reduce the chance of respiratory failure [7]. CT scans indicated significant improvement in the respiratory system (Fig. 1C). The diaphragmatic muscle showed a thickness of $0.22 \mathrm{~cm}$ and a diaphragmatic activity of $2.22 \mathrm{~cm}$. The MIP increased to $31.05 \mathrm{cmH}_{2} \mathrm{O}$. The patient was able to maintain a sitting position in a wheelchair to stay outdoors, to communicate with people for 30-40 minutes without difficulties. The patient and relatives were educated regarding the follow-up home-based rehabilitation activities (e.g. respiratory muscle and cough training). The time course of the case was briefly summarized in Fig. 2.

\section{Discussion}

Approximately two-thirds of patients with acute SCI experience complications such as atelectasis, pneumonia, and respiratory failure, which require mechanical ventilation $[8,9]$. Mechanical ventilation can adversely affect the structure and function of the diaphragm, so that weaning should start as soon as possible. The conservative management of respiratory dysfunction in SCI patients can be used to remove secretions and increase ventilation by various techniques, including postural drainage, percussion and vibration, assisted coughing, IMT, and noninvasive ventilatory support. IMT facilitates weaning for patients in ICU [10], however, its utility for ventilated SCI patients was rarely demonstrated. Different from common ventilated patients who developed ventilator-induced diaphragm dysfunction and lead to weaning difficulties, SCI patients mainly suffered from motor impairment and prolonged mechanical ventilation further impaired diaphragm function. Shimizu et al. reported a case where a closed-loop ventilation mode helped weaning from mechanical ventilation in an SCI patient [11]. However, it was a choice due to shortness in medical staffs. For patients with weaning delay and failure, rehabilitation and home mechanical ventilation center demonstrated favorable short-term and long-term clinical outcomes [12]. A comprehensive program requires cooperation of clinical staff from various disciplines, including physiatrist, physical and respiratory therapists, clinic psychologist, pulmonologist, 
intensivist and rehabilitation nurse [3].

In the present case, we demonstrated a tailored rehabilitation program throughout the weaning process. Starting from diagnosis and program design, we identified that patient's ventilation had a positive response to deep breaths with the help of EIT (Fig. 1B). Based on this information, we developed a program that covered passive activities and active training. Although EIT is a novel imaging technique and its potential in guiding weaning strategies was proposed [13], the present case is the first documented one (to our knowledge) using EIT to facilitate a rehabilitation program. Given the realtime visualization of the ventilation distribution, EIT may also play a role as live feedback during respiratory muscle training of lung function rehabilitation. Gundogdu et al. had implemented a rehabilitation program for SCI patients on a much large scale [3]. The program was developed by combining various methods published previously. In the present study, our therapy focused on airway clearance during the period of acute respiratory failure (including assisted coughing, postural drainage, active cycle of breathing technique, vibration, pat). At a later phase after pneumonia had been cured, the therapy mainly focused on IMT. After successful weaning, the therapy focused on increase cough peak flow, including assisted coughing and vibration maneuver. In addition to the rehabilitation program, more bedside tools were applied (e.g. ultrasound and EIT) to monitor the improvement of respiratory functions throughout the whole treatment period. The length and intensity were individualized not only through clinical personals but also through the patient himself via live feedbacks (e.g. the IMT and EIT devices). Physical excises might be useful at a later stage of rehabilitation treatment. We have also implemented a large amount of physical excises into our rehabilitation program and considered it helpful. Further prospective clinical trial needs to be conducted to prove the utility of such individual program.

\section{Conclusion}

Rehabilitation program specifically targeting the respiratory muscles would facilitate the weaning process for complete cervical spinal cord injury from the mechanical ventilator.

\section{ABBREVIATIONS}

$\mathrm{SCI}=$ spinal cord injury, $\mathrm{C}=$ cervical, $\mathrm{T}=$ thoracic, $\mathrm{L}=$ lumbar, $\mathrm{SBT}=$ spontaneous breathing trial, $\mathrm{PaO}_{2}=$ partial pressure of oxygen, $\mathrm{PaCO}_{2}$ = partial pressure of carbon dioxide, $\mathrm{FiO}_{2}$ $=$ fraction concentration of oxygen in inspired air, EIT = electrical impedance tomography, IMT = inspiratory muscle training, ICU = intensive care unit.

\section{ACKNOWLEDGEMENTS}

This study was supported by grants of Beijing Rehabilitation Hospital (2018-05).

\section{CONFLICT OF INTEREST}

Authors report no conflict of interest.

\section{AUTHOR CONTRIBUTIONS}

$\mathrm{HJ}$ and $\mathrm{ZZ}$ analysed the data and drafted the manuscript. BZ designed the study and revised the manuscript critically. $\mathrm{CZ}$ and YL collected the data and revised the manuscript critically. All authors approved the final version of manuscript.

\section{ETHICS APPROVAL AND CONSENT TO PARTICIPATE}

The study was approved by the Ethics Committee in Beijing Rehabilitation Hospital of Capital Medical University (Y20180012). Written informed consent was obtained from the parents of patient for the publication of this study.

\section{REFERENCES}

[1] Füssenich W, Hirschfeld Araujo S, Kowald B, et al. Discontinuous ventilator weaning of patients with acute SCI. Spinal Cord. 2018;56:461468.

[2] Arora S, Flower O, Murray NP, et al. Respiratory care of patients with cervical spinal cord injury: a review. Crit Care Resusc. 2012;14:64-73.

[3] Gundogdu I, Ozturk EA, Umay E, et al. Implementation of a respiratory rehabilitation protocol: weaning from the ventilator and tracheostomy in difficult-to-wean patients with spinal cord injury. Disabil Rehabil. 2017;39:1162-1170.

[4] Paternostro-Sluga T, Grim-Stieger M, Posch M, et al. Reliability and validity of the Medical Research Council (MRC) scale and a modified scale for testing muscle strength in patients with radial palsy. J Rehabil Med. 2008;40:665-671.

[5] Zachovajeviene B, Siupsinskas L, Zachovajevas P, et al. Effect of diaphragm and abdominal muscle training on pelvic floor strength and endurance: results of a prospective randomized trial. Sci Rep. 2019;9:19192.

[6] Sutt AL, Caruana LR, Dunster KR, et al. Speaking valves in tracheostomised ICU patients weaning off mechanical ventilation-do they facilitate lung recruitment? Crit Care. 2016;20:91.

[7] Zhu Y, Yin H, Zhang R, et al. High-flow nasal cannula oxygen therapy versus conventional oxygen therapy in patients after planned extubation: a systematic review and meta-analysis. Crit Care. 2019;23:180.

[8] Brown R, DiMarco AF, Hoit JD, et al. Respiratory dysfunction and management in spinal cord injury. Respir Care. 2006;51:853-868.

[9] Shavelle RM, DeVivo MJ, Strauss DJ, et al. Long-term survival of persons ventilator dependent after spinal cord injury. J Spinal Cord Med. 2006;29:511-519.

[10] Elkins M, Dentice R. Inspiratory muscle training facilitates weaning from mechanical ventilation among patients in the intensive care unit: a systematic review. J Physiother. 2015;61:125-134.

[11] Shimizu S, Nakajima M, Yamazaki M, et al. Weaning from longterm mechanical ventilation utilizing closed-loop ventilation mode (IntelliVent ${ }^{\circledR}-\mathrm{ASV} 囚$ ) in a patient with spinal cord injury. Spinal Cord Ser Cases 2018;4:51.

[12] Galeiras Vázquez R, Rascado Sedes P, Mourelo Fariña M, et al. Respiratory Management in the Patient with Spinal Cord Injury. Biomed Res Int. 2013;2013:168757.

[13] Zhao Z, Peng SY, Chang MY, et al. Spontaneous breathing trials after prolonged mechanical ventilation monitored by electrical impedance tomography: an observational study. Acta Anaesthesiol Scand. 2017;61:1166-1175. 
How to cite this article: Bin Zhang, Hongying Jiang, Chenxi Zhang, Yi Li, Zhanqi Zhao. Pulmonary Rehabilitation throughout the Weaning from Mechanical Ventilation for Complete Cervical Spinal Cord Injury: A Case Report. Signa Vitae. 2020;16(2):210-214. doi:10.22514/sv.2020.16.0058. 